\title{
Fabrication and Thermophysical Characterization of Nano-Porous Silica-Polyurethane Hybrid Aerogel by Sol-Gel Processing and Supercritical Solvent Drying Technique
}

\author{
Tae-Jin Yim, Sun Young Kim and Ki-Pung Yoo \\ Department of Chemical Engineering, Sogang University, C.P.O. Box 1142, Seoul 121-742, Korea \\ (Received 31 July $2001 \cdot$ accepted 20 September 2001)
}

\begin{abstract}
Thermally and mechanically enhanced nanoporous silica-polyurethane hybrid aerogel was synthesized by sol-gel processing and low temperature supercritical $\mathrm{CO}_{2}$ drying. A partially condensed silica solution and a polymeric MDI were used as the raw materials with a tertiary amine as a catalyst and 1,4-dioxiane as a dilution solvent. After the gelation reaction was completed, aged wet hybrid aerogel was dried by a low temperature supercritical $\mathrm{CO}_{2}$ drying technique. Also, thermophysical characteristics such as density, BET surface area, and thermal conductivity as a function of catalyst ratio and aging time of the synthesized hybrid aerogel were analyzed. It was found that, at a fixed target density, the lowest average pore size of the aerogel, $8 \mathrm{~nm}$, was obtained when the catalyst ratio was 0.1 wt.\%. Also, at these conditions, the BET surface area showed the highest surface area, $287.3 \mathrm{~m}^{2} / \mathrm{g}$. It was found that with decreasing average pore size and with increasing BET surface area, thermal conductivity tends to decrease. At pressure 1 torr, the sample aerogel showed the lowest thermal conductivity, $0.0184 \mathrm{~W} / \mathrm{mK}$.
\end{abstract}

Key words: Aerogel, Silica-Polyurethane Hybrid Aerogel, Supercritical Drying, Porous Material, Thermal Insulation

\section{INTRODUCTION}

Inorganic as well as organic aerogels are the lightest man-made ultraporous solid materials that are composed of randomly arrayed nanometer-size open pores. An inorganic aerogel was synthesized by Kistler [Kistler, 1931] in 1930 for the first time by sol-gel processing and supercritical fluid drying of water glass. Since then, numerous investigators have explored various synthetic methods with different types of starting materials such as tetramethyl orthosilicate, tetraethyl orthosilicate or polymeric substances.

To obtain a high-quality low-density transparent aerogel, two steps of sol-gel processing routes are devised: In the first sol-gel processing step, a hydrolysis reaction proceeds under acidic condition. Then, in the second processing step, a polymeric condensation reaction under basic condition follows [Brinker et al., 1984]. However, it was pointed out that when one adopts this two-step sol-gel processing route, it is necessary to permit long gelation time and thus the degree of transparency is deteriorated. To overcome this shortcoming in the two-step sol-gel processing, the Lawrence Livermore National Laboratory (LLNL) has proposed an improved two-step sol-gel processing method [Tillotson et al., 1988]. In this improved method, the by-product alcohol in the liquid residue after the hydrolysis was removed by distillation and subsequently a condensed silica solution was obtained. Then, in this condensed solution, a basic catalyst and diluting solvent was added and the gelation reaction proceeded to reach wet silica gel. This improved two-step sol-gel processing method was followed to obtain the condensed silica solution in the present study.

The next step to obtain the dried silica aerogel is to remove all the residue liquid in the wet gel. To do this, two supercritical fluid drying (SFD) methods can be utilized: one is the low-temperature

†T whom correspondence should be addressed.

E-mail: kpyoo@ccs.sogang.ac.kr
SFD using liquid state $\mathrm{CO}_{2}$ [Henning et al., 1981] and the other is high-temperature SFD utilizing organic solvent [Tewari et al., 1985]. When the low-temperature SFD is adopted, the surface and internal pores of the dried aerogel are hygroscopic and thus an additional surface stabilization step is necessary to convert the surface as the hydrophobic [Lee et al., 1995; Yokogawa et al., 1995]. The surface treatment process is to convert - $\mathrm{OH}$ groups on the surface to - R groups [Iler, 1978].

On the other hand, organic aerogels such as RF (Resorcinol-Formaldehyde) and MF (Melamine-Formaldehyde) aerogels can be synthesized by a similar synthetic route as the case of silica aerogel. These organic aerogels also show a high-degree of nano-porosity as the case of silica aerogel. Physicochemical examination of the thermal characteristic behaviors of organic aerogels was made by the present authors [Kim et al., 2001], who found that organic aerogels such as RF aerogel show extremely low thermal conductivity than any other man-made functional materials. Furthermore, a method of synthesizing polyurethane-base organic aerogel was proposed from polymeric Diphenylmethane-4,4-Diisocyanate (MDI) [Biesmans et al., 1998].

Due to the nano-porosity with full of nanometer pores, any type of aerogel possesses several intriguing thermophysical characteristics. For example, an aerogel, whether it is organic or inorganic, possesses the lowest refractive index, dielectric constants, sonic velocity and thermal conductivity among man-made solid substances. The various types of aerogels are widely used as catalyst substrates, super thermal insulation materials, and elementary particle acceleration device material. Also, in recent years, organic and thermally pyrolyzed carbon aerogels have been used for super capacitors, electrodes for capacitive deionization and thermal energy storage material [Hrubesh, 1998].

In general, aerogels can be classified into two types as described above: inorganic aerogels such as silica aerogel and organic aerogels such as resorcinol-formaldehyde aerogel. Inorganic aerogel has 
an advantage of thermal stability and low thermal conductivity. However, it is mechanically fragile. In the case of organic aerogel, it is mechanically there is an intrinsic limitation for finding commercial utilities that consume a large amount of each type of aerogel. Fundamentally, if someone can find a synthetic route to reach a hybrid aerogel that is composed of inorganic and organic aerogels, it might resolve the intrinsic limitation of thermal and mechanical weakness in each of organic or inorganic aerogel. Instead, the hybrid aerogel can possess the advantageous aspects from the two different types of aerogels. These considerations are the starting point of the present work. In recent years, the present authors have been placing their attention on synthesizing a hybrid aerogel of silica and polymeric aerogel.

In the present work, a chemical reaction route of obtaining a hybrid wet gel was devised by bonding the organic functional groups in polymeric MDI with tetramethyl orthosilicate (TMOS) that is the feed material to get silica aerogel. The organic part in the hybrid aerogel facilitates the control of the micropore structures and makes hydrophobic behavior. At the same time, the inorganic part in the hybrid aerogel enhances thermal and dimensional stability [Schwertfeger et al., 1992]. Various types of hybrid aerogels were obtained by the reaction of silanol and isocyanate under various synthetic conditions. Also, the thermophysical properties such as BET surface area, pore size distribution, and thermal conductivity were measured.

\section{SYNTHESIS SILICA-POLYURETHANE HYBRID AEROGEL}

\section{Sol-Gel Processing of Polymeric MDI and TMOS}

In general, sol-gel processing is a step to fabricate wet gel from sol solution by the addition of appropriate catalyst, diluent, and subsequent aging. The internal structure of wet gel is cross linked and branched open pores in a random manner. In the present study, the sol solution was synthesized by adding a certain amount of water and catalyst into tetramethyl orthosilicate (TMOS) with continuous stirring of the solution. Then, after the produced alcohol was removed by distillation, condensed silica solution, that was partially hydrolyzed and condensed, was obtained [Tillotson et al., 1992]. Finally the hybrid wet gel for further aging was obtained by adding polymeric MDI and catalyst to the partially condensed silica solution. As a catalyst, 2-dimethylaminoethanol, which is one of tertiary amines and as a diluent, 1,4-dioxane was used.

\section{Supercritical Solvent Drying}

Two intrinsic problems in the process of aging wet gel and subsequent drying wet gel are how to prevent shrinking during aging step and cracking internal pores of aerogel during the drying step. In general, the shrinking phenomenon is due to several reasons such as gelation reaction and occurrence of osmotic forces during the solvent substitution reaction. Other reasons include the different capillary forces between different micropore size distribution and existence of a meniscus between gas and liquid phase in the wet gel accelerate shrinking and cracking [Brinker et al., 1990]. Among them, effect of shrinking during the gelation reaction is insignificant when compared to other factors. The osmotic effect arises due to the competitive chemical diffusion by the concentration gradient of species during the substitution of hydrophilic diluent solvent by $\mathrm{CO}_{2}$-philic solvent. The existence of osmotic behavior in the pro- cess of substitution of these two solvents in the wet gel accelerates shrinking. Also, one of the major shrinking effects is due to the capillary force difference during the liquid-gas phase transition during drying.

In the inside of the wet gel, liquid consisting of added diluent solvent and unreacted feed substances exists. In principle, the residue liquid in the wet gel can be removed by conventional evaporation and the supercritical drying method. In case of evaporative drying, the gas-liquid meniscuses in every micropore is propagated into the inside of the pores. Thus, when the pore size distribution is not uniform, there occurs capillary pressure difference, force difference $\Delta \mathrm{P}$ among different pores. Since the capillary surface tension is maintained by a solid, the different size distribution of pores gives rise to the difference of surface tension. That is,

$$
\Delta \mathrm{P}=\frac{-2 \gamma \cos \theta}{\mathrm{d}}
$$

where $\gamma$ denotes surface tension, $\theta$, the contact angle, $d$, the pore diameter and $\Delta \mathrm{P}$ is the capillary surface tension. Thus, when one adopts evaporative drying to reach aerogel from aged wet gel, the shrinking and cracking of the internal solid pore network cannot be prevented. Also, it takes a tremendous amount of time to complete evaporative drying. Therefore, the gel obtained through solvent evaporation incurs serious shrinking and becomes xerogel of high density.

Alternatively if one can adopt the supercritical solvent drying of wet gel, liquid in the micropores can be converted into gas by a continuous phase transition. Specifically the liquid phase is transformed into supercritical phase and subsequently transformed into gas phase without any type of meniscus appearing between liquid and gas phase. Thus, there is no occurrence of the capillary force difference among different pore size distributions because there is no sudden phase transition from liquid to gas phase. Obviously, one can remove liquid from micropores of the wet gel without giving rise to the shrinking and cracking of the pores. In general, the supercritical solvent drying method can be arbitrarily classified by high temperature drying and low temperature drying depend on what kind of liquid to be removed from the wet gel.

The major component of the residual liquid in wet gel is water and diluent solvent. The critical point of water $(\mathrm{Tc}=647.3 \mathrm{~K}, \mathrm{Pc}=$ $22 \mathrm{MPa}$ ) is extremely high and if one intends to convert water into supercritical state, the silica solid pore network can be damaged due to the employment of high temperature. Thus, before supercritical drying, it is necessary to replace water in wet gel by other solvent that retains mild condition of the critical point. Traditionally, organic solvents such as alcohols and acetone are used frequently to substitute water to organic solvent before drying. Then, the organic liquid can be removed from pores of wet gel by the high temperature drying. Thus, to carry out high temperature drying, it is necessary to substitute liquid in the pores by alcohol or acetone. One of the advantages of the high temperature drying is that the solid surface of the gel can transform into the hydrophobic and be free from the limitation of shrinking. However, the high temperature drying method uses flammable organic solvents which makes this method exposed to the possibility of fire and explosion hazards when there is any sort of leakage during operation. Besides, it cannot be free from the possibility of rearrangement of gel network in high temperature drying method. For example, methanol 
was used frequently in the high temperature drying. The critical temperature of this substance is $240^{\circ} \mathrm{C}$ and critical pressure is $7.82 \mathrm{MPa}$. Thus, when one uses methanol, a usual drying condition should be above $300{ }^{\circ} \mathrm{C}$, and 13.0 MPa.

Alternatively, to eliminate possible fire hazard in the high temperature supercritical solvent drying, a low temperature supercritical drying method can be adopted. In the case of low temperature supercritical solvent drying, carbon dioxide is used as a substitute solvent instead of organic solvent such as ethanol. However, when one adopts liquid carbon dioxide, caution must be taken about whether there can arise liquid-liquid contact phases between liquid carbon dioxide and residue liquid which are immiscible with each other during the drying step. When there exists any substance which tends to immiscible with liquid carbon dioxide, there can be also exist a different force of surface tension among different pore sizes, and it gives rise to cracking the wet gel during the low temperature supercritical drying. Thus, to eliminate the possibility of cracks, it is necessary to replace liquid in the wet gel by $\mathrm{CO}_{2}$-philic solvent before carrying out low temperature drying. While the low temperature drying with carbon dioxide has the advantage of freeing from fire and explosion hazards, one unfavorable thing is the shrinking phenomenon more than the case of high temperature operation. Also, there exist $-\mathrm{OH}$ groups on the aerogel surface; therefore, the aerogels via low temperature drying become hydrophilic. Thus, if one exposes the aerogel after the drying step into air, it is apt to severe shrinking and cracks due to rapid humidification of the hydrophilic aerogel. Thus, this low temperature method is not useful for drying wet gel in that it becomes a stable aerogel from shrinking.

\section{Fabrication of Polyurethane-Silica Hybrid Aerogel}

The hybrid aerogel was synthesized with silica and polyurethane as in the following processes. First, tetramethyl orthosilicate (TMOS), water, and $\mathrm{HCl}$ were added into a beaker by the molar ratio of 1 : $1.3: 10^{-5}$. Then, this feed solution was mixed by stirrer for an hour. Second, produced alcohol was removed from this feed solution by a batch distillation. Third, the feed sol solution was partially hydrolyzed to obtain condensed silica solution [Tillotson et al., 1992]. In this partially condensed solution (CS), polymeric MDI solution was added by varying density and the amount of catalyst to reach silicaorganic hybrid aerogel. The hybridization reaction proceeded in this step. However, it is not possible to evaluate quantitatively the density and structure of the hybrid wet gel. Thus we assumed that the condensed silica solution was completely hydrolyzed and the number of $-\mathrm{OH}$ groups in

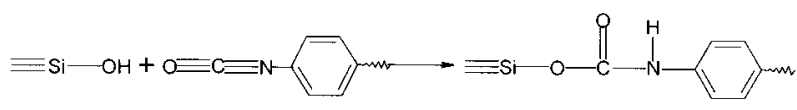

silanol was assumed as 4 before reaction of isocyanate. Also, in the present study, target density was defined as the consumed polymeric MDI and condensed silica solution with respect to the total volume of solution consumed. The 1,4-dioxane was used as a dilution solvent. In the hybridization step, 2-dimethylaminoethanol, which is used in the polyurethane forming process, was used as the catalyst. This catalyst, which is one of basic tertiary amine, assists to form a complicated intermediate by acting as an electron donor to the carbonyl carbon in isocyanate [Woods, 1990]. The catalyst substantially affects the pore size and surface area of hybrid aerogel [Brinker et al., 1990]. The catalyst ratio was defined by Eq. (3). Then, the morphological change of gel was examined by varying the catalyst ratio.

$$
\text { catalyst ratio }=\frac{\text { catalyst mass }}{[\mathrm{CS}+\text { Polymeric MDI }+ \text { diluent }+ \text { catalyst }] \text { mass }}
$$

Since the completion of the gelation of the hybrid aerogel takes about a week, the wet gel was aged for a couple of weeks. Then, low temperature supercritical $\mathrm{CO}_{2}$ drying was performed. In the present work, a drying of wet gel carried out by the following steps. In the first place, wet gel was placed into the autoclave drier. Then, liquid $\mathrm{CO}_{2}$ was slowly fed into the drier while drier pressure was maintained up to $10.0 \mathrm{MPa}$ and progressively liquid inside the gel pores was exchanged by liquid $\mathrm{CO}_{2}$. Next, the drier was heated to $37{ }^{\circ} \mathrm{C}$ to make supercritical state of $\mathrm{CO}_{2}$. Then, the drier was slowly evacuated by purging out the supercritical $\mathrm{CO}_{2}$ by the $5 \mathrm{~L} / \mathrm{min}$ flow rate.

\section{RESULTS AND DISCUSSION}

\section{Revised Low Temperature Supercritical $\mathrm{CO}_{2}$ Drying}

Low temperature supercritical $\mathrm{CO}_{2}$ drying was devised by the Lawrence Berkeley Laboratory (LBL) [Tewari et al., 1986]. However, as summarized in Table 1, the LBL method adopts rather complicated drying steps of the low temperature supercritical $\mathrm{CO}_{2}$ drying. After scrutiny of the LBL idea, the present authors proposed a simplified low temperature drying technique while maintaining completely equivalent drying performance. The idea was summarized in Table 1 together with the LBL method.

\section{FTIR Analysis of Hybrid Aerogel}

The functional group characteristics of silica, polymeric MDI and the hybrid aerogel by synthesizing these materials were analyzed by FTIR (MIDAC, PRS) and the results were summarized in Fig. 1. Also, the functional groups in accordance with the wave number described by circled number in Fig. 1 were summarized in

Table 1. Low temperature SC $\mathrm{CO}_{2}$ drying of present work and LBL method

\begin{tabular}{ll}
\hline \hline Steps of the Lawrence Berkeley Laboratory method & Steps of the new method devised in the present study \\
\hline $\begin{array}{l}\text { 1. Liquid in wet gel is substituted by ethanol } \\
\text { (it takes several days) }\end{array}$ & 1. Place wet gel into autoclave without substituting solvent \\
$\begin{array}{ll}\text { 2. Placing excess amount of ethanol and wet gel into autoclave } & \text { 2. Substitute liquid in wet gel by liquid } \mathrm{CO}_{2} \\
\text { 3. Replacing excess amount of ethanol by liquid } \mathrm{CO}_{2} & \text { 3. Carry out supercritical } \mathrm{CO}_{2} \text { drying } \\
\text { 4. Substitute liquid in wet gel by liquid } \mathrm{CO}_{2} & \text { 4. Depressurization } \\
\text { 5. Perform supercritical } \mathrm{CO}_{2} \text { drying } & \\
\text { 6. Depressurization } & \end{array}$ \\
\hline
\end{tabular}




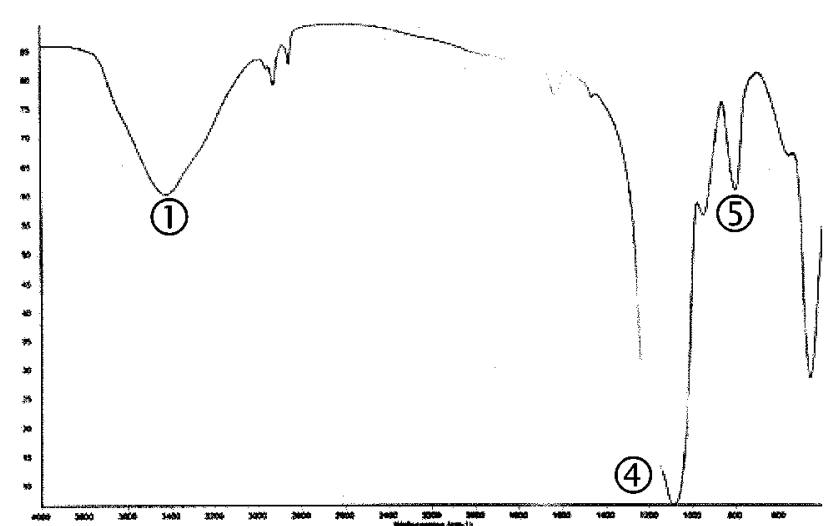

(a) silica aerogel

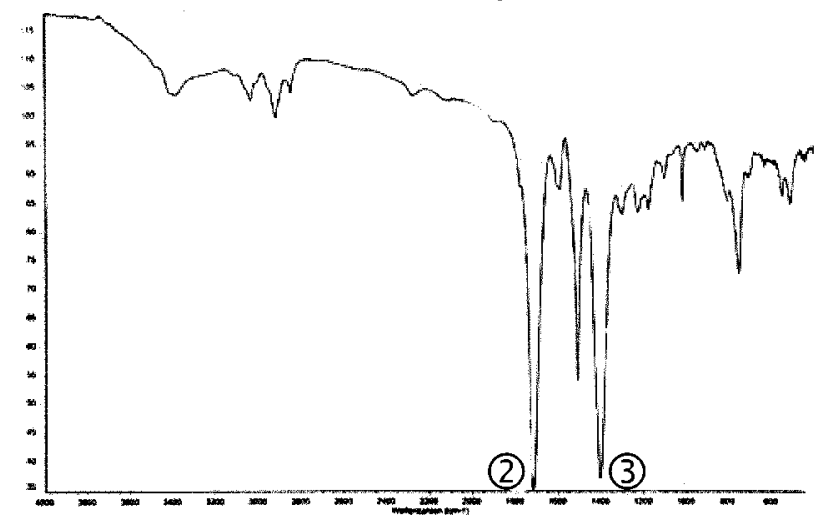

(b) polymeric MDI aerogel

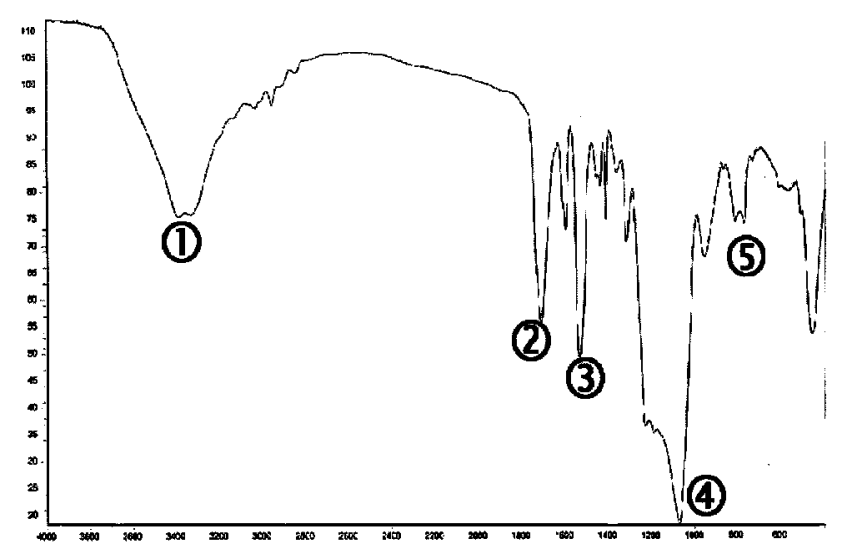

(c) hybrid aerogel

Fig. 1. FTIR spectra of aerogels.

Table 2. In Fig. 1, the circled numbers (1), (4) and (5) represent the inorganic groups in silica aerogel. Also, the numbers (2) and (3) are the organic groups in polymeric MDI-based organic aerogel.

If there exist unreacted organic and inorganic groups, they will not form gel. These unreacted residues will be removed from the gel during the supercritical drying process. In the case of hybrid aerogel, however, it was found that it contained all possible functional groups from (1) to (5) in the final structure. Thus it can be considered that the hybrid aerogel was formed through the chemical reaction bond formation among inorganic and organic gel materials.

3. Analysis of Pore Size Distribution and BET Surface Area

The variation of average pore sizes examined by the BET ana-
Table 2. Wave numbers corresponding to each functional groups

\begin{tabular}{ccl}
\hline \hline Number & $\begin{array}{c}\text { Wave number } \\
\left(\mathrm{cm}^{-1}\right)\end{array}$ & Functional group \\
\hline (1) & 3500 & $\mathrm{H}$ of silanol \\
$(2)$ & 1700 & $\mathrm{C}=\mathrm{O}$ of NCO \\
(3) & 1400 & $\mathrm{C}-\mathrm{N}$ of NCO \\
(4) & 1100 & $\mathrm{Si}-\mathrm{O}-\mathrm{Si}$ (asymmetric) \\
(5) & 800 & $\mathrm{Si}-\mathrm{O}-\mathrm{Si}$ (symmetric or vibration \\
& & mode of ring) \\
\hline
\end{tabular}

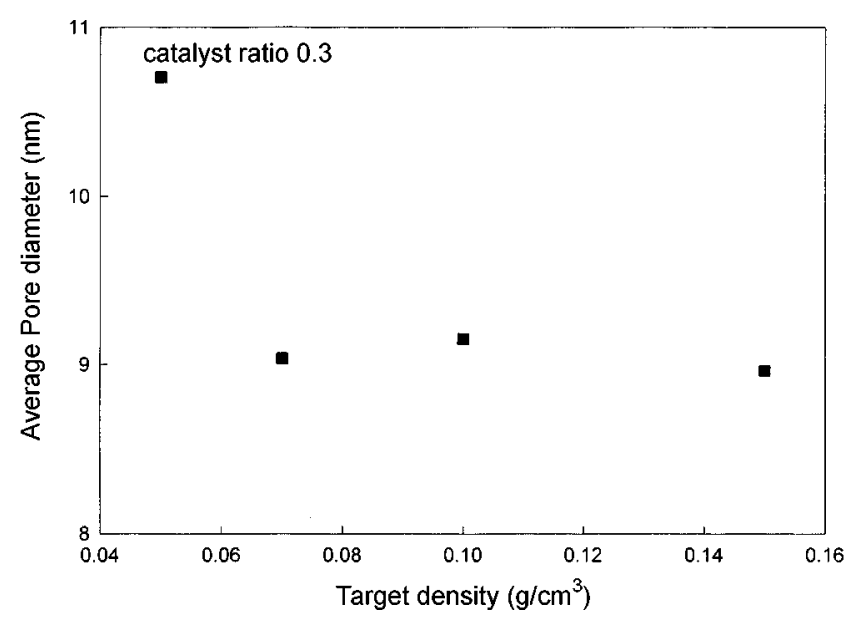

Fig. 2. Average pore diameter with respect to the variation of target density.

lyzer with respect to the variation of the target density is shown in Fig. 2. It was found that there is no noticeable variation of the average pore sizes when the target density lies above $0.06 \mathrm{~g} / \mathrm{cm}^{3}$. Also, the variation of average pore sizes of the aerogel with respect to the catalyst ratio is shown in Fig. 3. Upon the results shown in Fig. 3 , it was found that the average pore sizes tend to decrease with increasing the catalyst ratio at the low catalyst ratio region. However, when the catalyst ratio reaches around 0.1 , the average pore sizes show the lowest values and again start to increase with increasing the catalyst ratio. These trends tell us that when one sim-

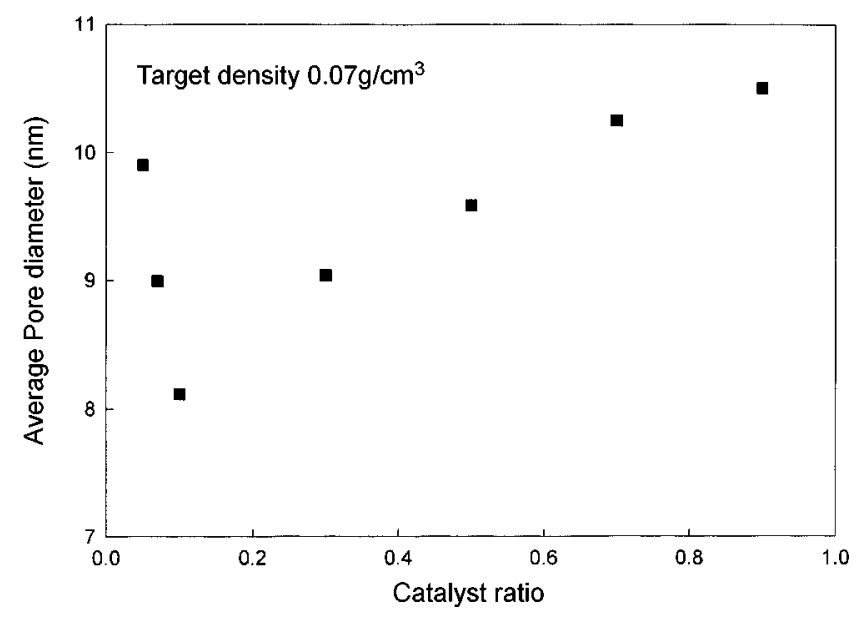

Fig. 3. Average pore diameter with respect to the variation of catalyst ratio. 
ply varies the density of gel by adding diluents, the variation of density cannot give variation effect of pore sizes. However, when the catalyst ratio was varied and had an effect on the chemical reaction rate of gelation, the average pore sizes tended to vary sensitively with the variation of the catalyst ratio. Therefore, it can be con-

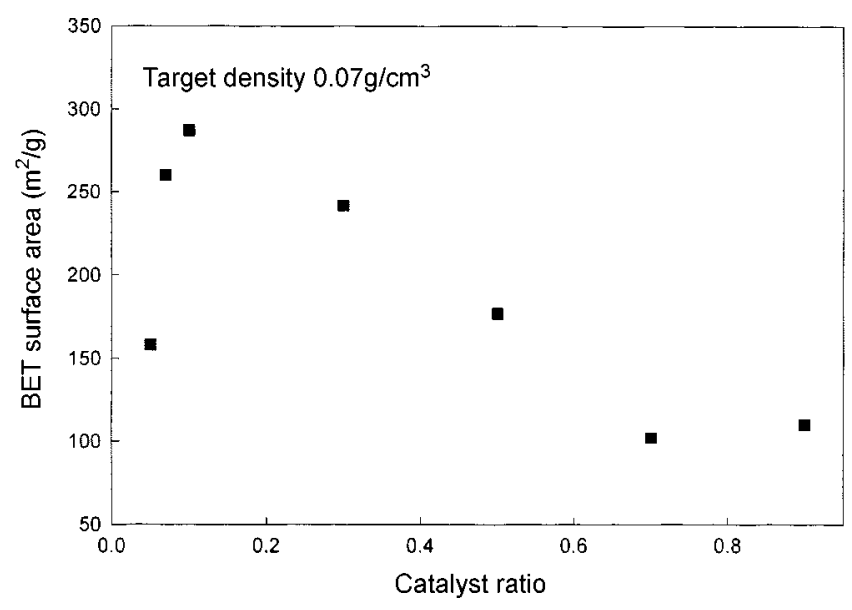

Fig. 4. BET surface area with respect to the variation of target density.

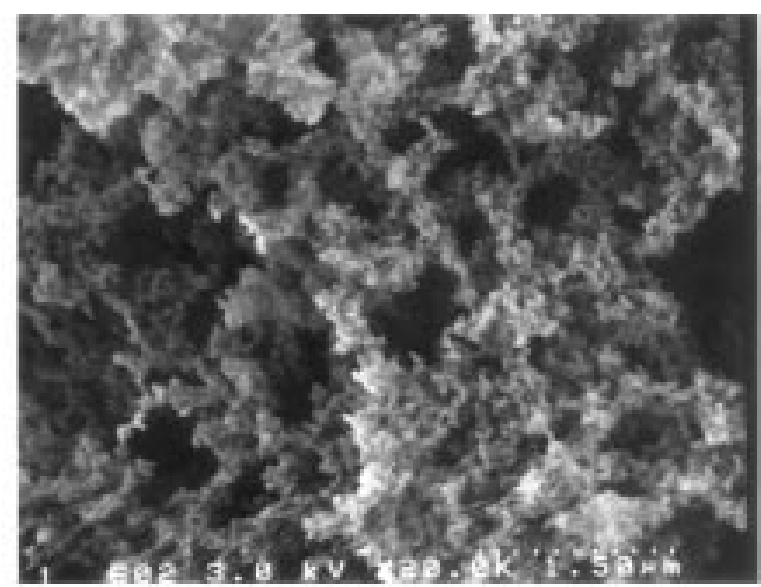

(a) The catalyst ratio is 0,1 and the target density is $0.07 \mathrm{~g} / \mathrm{cm}^{3}$

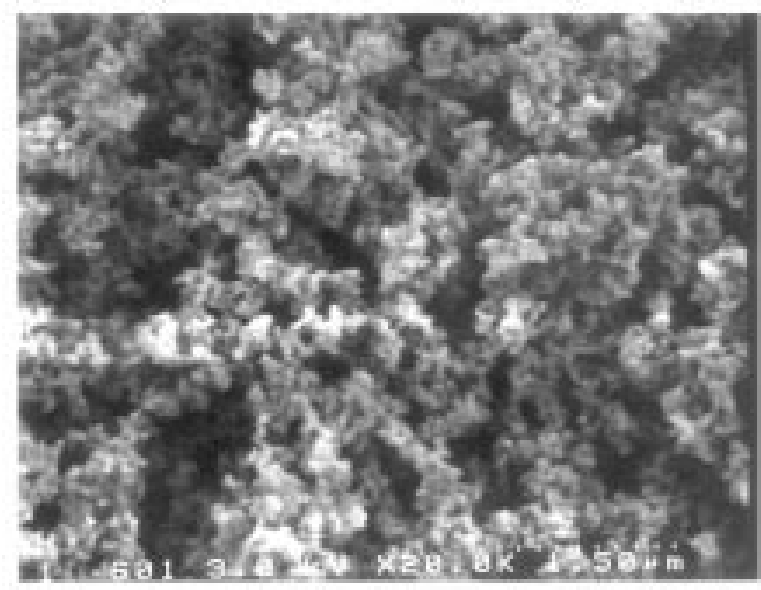

(b) The catalyst ratio is 0.9 and the target densily is $0.07 \mathrm{~g} / \mathrm{cm}^{3}$

Fig. 5. SEM photographs of hybrid aerogel at constant target density. cluded that the catalyst ratio is the most important synthetic parameter to vary the internal pore size distribution of the final hybrid aerogels. Again, the variation of the specific surface area of aerogel with respect to the catalyst ratio is shown in Fig. 4. According to this result, the hybrid aerogel with the maximum specific surface area, $287.3 \mathrm{~m}^{2} / \mathrm{g}$, can be accomplished at the catalyst ratio 0.1 which can bring the smallest average pore size distribution.

The SEM photographs of hybrid aerogels obtained at the catalyst ratio 0.1 and 0.9 at the same density, $0.07 \mathrm{~g} / \mathrm{cm}^{3}$ are shown in Fig. 5. The SEM photographs show that the case of catalyst ratio 0.1 was agglomerated with more fine particles than the case of the catalyst ratio 0.9 . The case of the catalyst ratio 0.9 shows more densely packed pore structures and less porous structure than the catalyst ratio 0.1 . As a result, we could make the low average pore sizes and large specific surface area of aerogel at catalyst ratio 0.1 and large average pore sizes and low specific surface area at the catalyst ratio 0.9 .

The experimental variation of the specific surface area with respect to the variation of the average pore size distribution of the hybrid aerogel is shown in Fig. 6. As one can see, it is clear that the BET surface area is found to be decreased with increasing the average

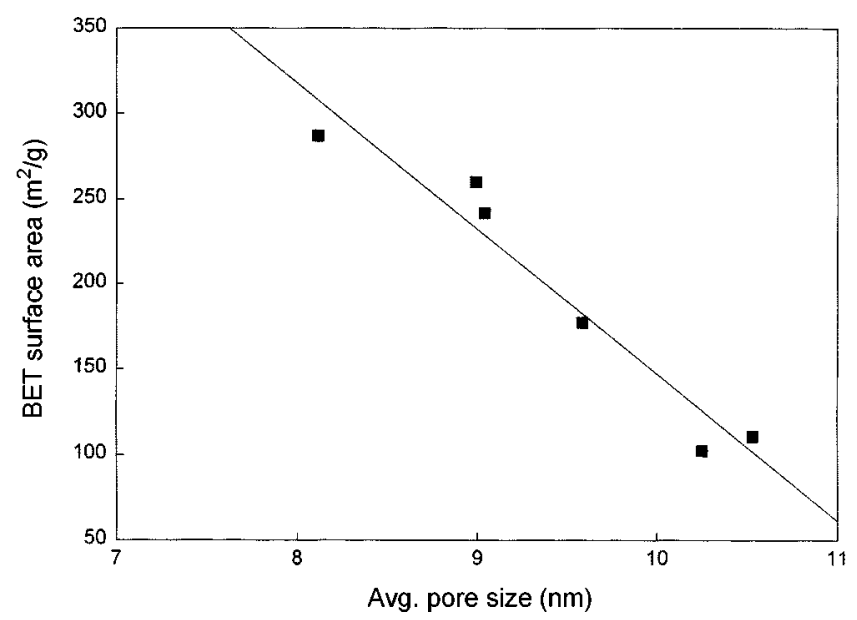

Fig. 6. Relation between average pore size and BET surface area.

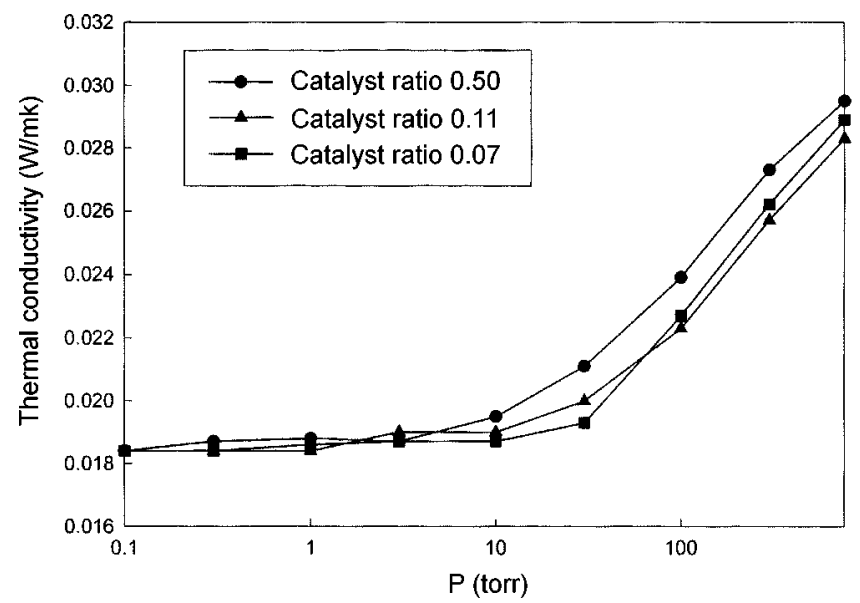

Fig. 7. Thermal conductivity with the variation of catalyst ratio at target density $0.07 \mathrm{~g} / \mathrm{cm}^{3}$. 


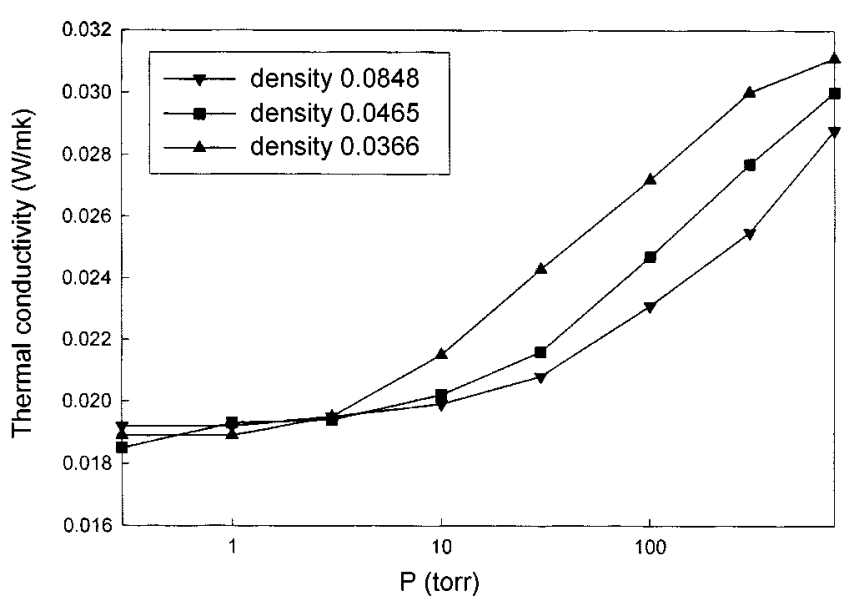

Fig. 8. Thermal conductivity with the variation of density at catalyst ratio 0.11 .

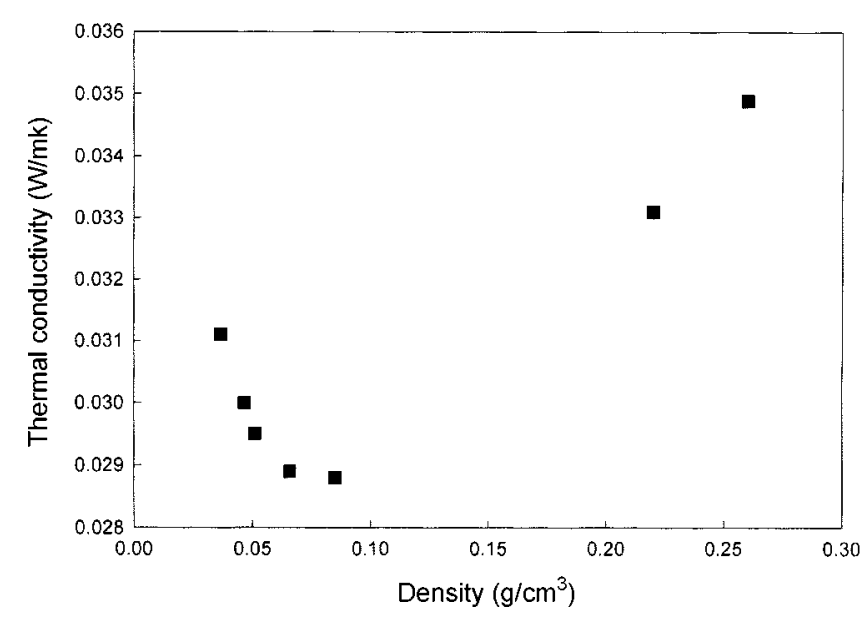

Fig. 9. Thermal conductivity at 760 torr.

pore sizes. This trend agrees with the general relation between the pore size distribution and the specific surface area. In this figure the experimental results of the surface area as a function of the pore size below the region of the catalyst ratio 0.1 were omitted due to the intrinsic uncertainties of the instruments.

\section{Analysis of Thermal Conductivity}

Here the thermal characteristics of the hybrid aerogels are discussed. The thermal conductivity was measured by a hot-wire transient analyzer [Carslaw et al., 1959]. This device was constructed by the present authors and its reliability was demonstrated in detail elsewhere [Grazzini et al., 1996]. In Fig. 7, the measured results of the thermal conductivity with respect to the variation of pressure at different catalyst ratios are summarized. It was found that the variation of the thermal conductivities with respect to the variation of

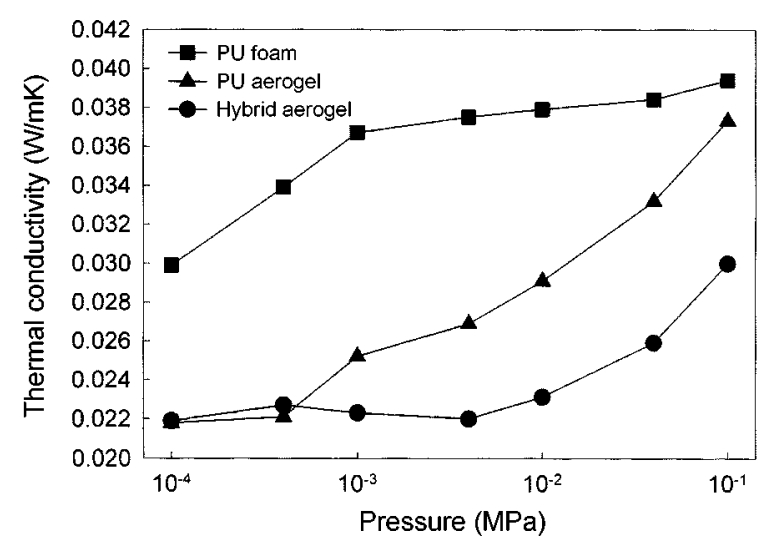

Fig. 10. Thermal conductivities of PU foam, polymeric MDI and hybrid aerogel.

the fine pore size distribution shows similar results regardless of the pore size change. In other words, the thermal conductivity does not become lowered with lowering the average pore sizes of the hybrid aerogel. However, the variation of the density can have significant effect on the thermal conductivity of aerogel as shown in Fig. 8. It was found that the thermal conductivity tends to decrease with decreasing the density of aerogel.

In Fig. 9, thermal conductivity of the aerogel as a function of density at an ambient pressure is shown. However, as one can see, the thermal conductivity tends to decrease in the low density region and inverts to increasing thermal conductivity in the high density region. In other words, like Table 3 , in the high density region above $0.1 \mathrm{~g} / \mathrm{cm}^{3}$ of the hybrid aerogel, the thermal conductivity tends to decrease with decreasing the target density. It means that the prime contribution on thermal conductivity of the aerogel is due to the characteristics of the solid structure. However, in the low density region below $0.1 \mathrm{~g} / \mathrm{cm}^{3}$, the major factor contributing to the thermal conductivity of the hybrid aerogel was the characteristics of gas species in the micropores. It also means that the contribution of the radiation is negligible.

In Fig. 10, the thermal conductivities measured by changing pressure at a fixed density, $0.05 \mathrm{~g} / \mathrm{cm}^{3}$ for samples such as PU foam, polymeric MDI and hybrid aerogel are shown. The inorganic/organic hybrid aerogel showed the lowest thermal conductivity. It is because the organic/inorganic hybrid aerogel tends to decrease the thermal conduction through the solid part than the nonporous solids.

In Fig. 11 are summarized the thermal conductivities of sample aerogels obtained by the catalyst ratio, 0.2 ; the target density $0.15 \mathrm{~g} /$ $\mathrm{cm}^{3}$; and aged for 2 and 4 weeks at $50{ }^{\circ} \mathrm{C}$, respectively. In general, when one carries out ageing the silica aerogel or organic aerogel, the mechanical strength of these tends to be strong due to the coagulation of fine particles. Thus, it is known that when the ageing

Table 3. Major factors affecting the overall thermal conductivity of the hybrid aerogel

\begin{tabular}{ll}
\hline \hline Low density region & High density region \\
\hline 1. Physical characteristics of gas species & 1. Physical characteristics of solid structure \\
$\begin{array}{l}\text { 2. Knudsen diffusion } \\
\text { 3. Density of aerogel: High density aerogel shows lower }\end{array}$ & $\begin{array}{l}\text { 2. Knudsen diffusion } \\
\text { thermal conductivity than low density aerogel }\end{array}$ \\
\hline
\end{tabular}




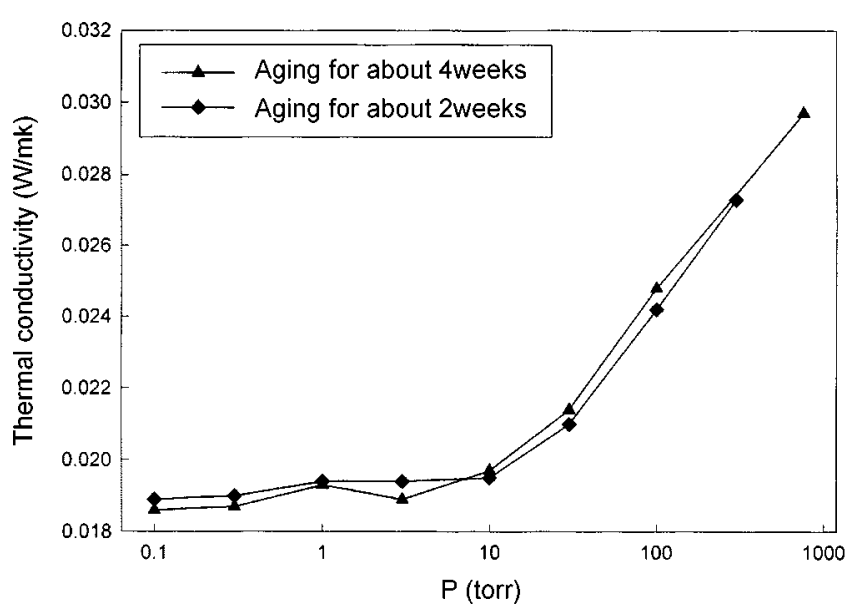

Fig. 11. Thermal conductivity variation with respect to aging times of wet gel.

is carried out for a long period of time, there is no significant shrinking of pores during the supercritical carbon dioxide drying, and it results in no significant increase of density during the drying process. As the result, the thermal conductivity of these aerogels stays low values. However, in the case of an organic/inorganic hybrid aerogel, there is no occurrence of such phenomena and it shows almost similar value of the thermal conductivities regardless of the ageing conditions. It seems that the inorganic functional groups in the silica are added into organic groups in the formation of hybrid aerogel, and it tends to increase intrinsically the mechanical strength and to prevent shrinking during the supercritical drying step. Also, these formation characteristics of the hybrid aerogel seem to make lower thermal conductivity than the case of hybrid aerogel formed by PU foam and polymeric MDI.

\section{Analysis of Humidity Absorption}

The hybrid aerogel obtained by the low temperature supercritical $\mathrm{CO}_{2}$ drying tends to hygroscopic behavior. Thus, when the aerogel is exposed to an open air, it absorbs water vapor and it can become fragile with a small mechanical shear force. The silica part of the hybrid aerogel contains hydrophilic $\mathrm{SiO}_{2}$ bonding structure and unbonded branch silanol group, $\mathrm{Si}-\mathrm{OH}$. The $-\mathrm{OH}$ group in this

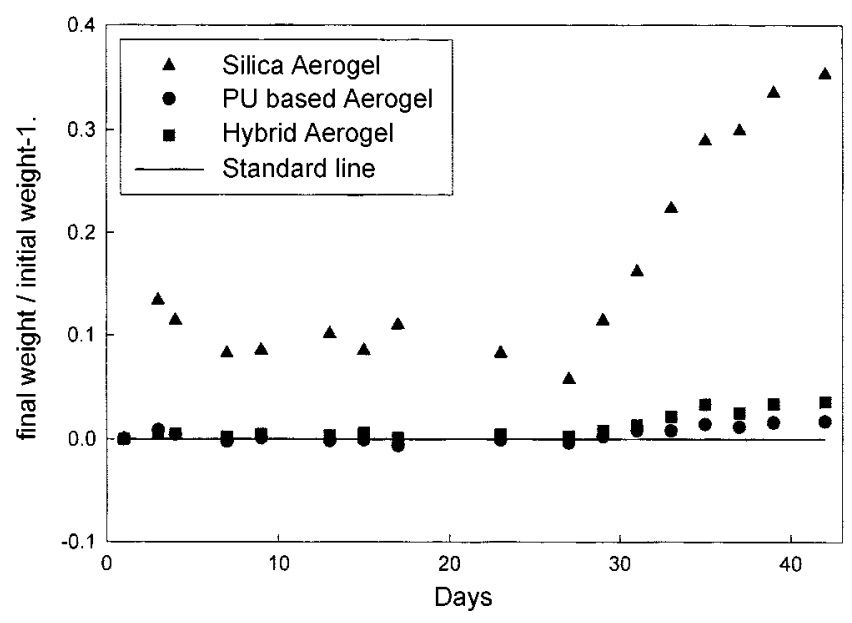

Fig. 12. Absorbance of moisture of aerogels. structure can form chemical bond with water and it can become hydrophilic aerogel. In this regard, attention has been placed on how to transform the hydrophilic silica aerogel to hydrophobic one. In Fig. 12, pure silica aerogel absorbs significant amount of water with time and there is a significant weight increase. On the contrary, the polyurethane aerogel is not absorbed water with time at all. In the case of silica-polyurethane hybrid aerogel, it is relatively stable to the exposure to humid air and it does not absorb water at all even for a long period of time. It is a target behavior of the hybrid aerogel.

\section{CONCLUSIONS}

By a condensed silica solution as an inorganic part and a polymeric MDI as an organic part, a new silica-polyurethane hybrid aerogel was synthesized. In the sol-gel processing reaction, a tertiary amine was used as a catalyst and 1,4-dioxiane was used as a dilution solvent. After a gelation reaction was completed, aged wet hybrid gel was dried by a low temperature supercritical $\mathrm{CO}_{2}$ drying technique. Also, thermophysical characteristics such as density, BET surface area, and thermal conductivity as a function of catalyst ratio were analyzed. It was found that, at a fixed target density, the lowest average pore size of the aerogel, $8 \mathrm{~nm}$, was obtained when the catalyst ratio was 0.1 . Also, at these conditions, the BET surface area showed the highest surface area, $287.3 \mathrm{~m}^{2} / \mathrm{g}$. It was found that with decreasing average pore sizes and with increasing the BET surface area, the thermal conductivity tends to decrease. At pressure 1 torr, the sample aerogel showed the lowest thermal conductivity, 0.0184 $\mathrm{W} / \mathrm{mK}$. In summary, gelation with respect to the variation of the catalyst ratio significantly affects the pore size distribution, and the variation of density greatly affects the thermal conductivity of the hybrid aerogel. One can envisage that this new silica-polyurethane hybrid aerogel obtained in the present study can be used for the purpose of thermal and sound insulation purposes with enhanced mechanical and thermal strength and any other existing porous materials.

\section{REFERENCES}

Biesmans, G., Randall, D., Francais, E. and Perrut, M., "Polyurethanebased Organic Aerogels Thermal Performance,' J. Non-Cryst. Solids, 225, 36 (1998).

Brinker, C. J., Drotning, D. W. and Scherer, G. W., "A Comparison Between the Densification Kinetics of Colloidal and Polymeric Silica Gels,' Better Ceramics Through Chemistry I, Brinker, C. J., Clark, D. E. and Ulrich, D. R., eds., North-Holland, New York (1984).

Brinker, C. J. and Scherer, G. W., "Sol-Gel Science,' Academic Press Inc., Boston (1990).

Carslaw, H. S. and Jaeger, J. C., "Conduction of Heat in Solids", $2^{\text {nd }}$ ed., Chap. 10, Oxford University Press, London (1959).

Grazzini, G., Balocco, C. and Lucia, U., "Measuring Properties with Parallel Wire Method,' Int. J. Heat Mass Transfer, 39(10), 2009 (1996).

Hrubesh, L. W., “Aerogel Applications,' J. Non-Cryst. Solids, 225, 335 (1998).

Iler, R. K., "Chemistry of Silica,' John Wiley \& Sons, New York (1978). Kim, S. Y., Yeo, D. H., Lim, J. W., Yoo, K.-P., Lee, K. H. and Kim, H. Y., 
"Synthesis and Characterization of Resorcinol-Formaldehyde Organic Aerogel,' J. Chem. Eng. Jpn, 34, 216 (2001).

Kistler, S. S., "Coherent Expanded Aerogels and Jellies,' Nature, 127, 741(1931).

Lee, K.-H., Kim, S.-Y. and Yoo, K.-P., "Low-density, Hybrophobic Aerogels,' J. Non-Cryst. Solids, 186, 18 (1995).

Schwertfeger, F., Glaubitt, W. and Schubert, U., "Hydrophobic Aerogels from $\mathrm{Si}(\mathrm{OMe})_{4} / \mathrm{MeSi}(\mathrm{OMe})_{3}$ Mixtures,' J. Non-Cryst. Solids, 145, 85 (1992).

Tillotson, T. M. and Hrubesh, L. W., 'Transparent Ultralow-density Silica Aerogels Prepared by a Two-step Sol-gel Process,' J. Non-Cryst. Solids, 145, 44 (1992).

Tillotson, T. M., Hrubesh L. W. and Thomas, I. M., "Partially Hydro- lyzed Alkoxysilanes as Precursors for Silica Aerogels,' Better Ceramics Through Chemistry III, Brinker, C. J., Clark, D. E. and Ulrich, D. R., eds., MRS, Pittsburgh (1988).

Tewari, P. H. and Arlon, J. H., "Process for Forming Transparent Aerogel Insulating Arrays,' US Patent 4610863 (1986).

Tewari, P. H., Hunt, A. J. and Loffuts, K. D., "Advance in Production of Transparent Silica Aerogels for Window Glazing,' Proceedings of the $1^{\text {st }}$ International Symposium on Aerogels, Wurzburg (1985).

Woods, G., "The ICI Polyurethanes Book,' $2^{\text {nd }}$ ed., ICI Polyurethanes (1990).

Yokogawa, H. and Yokoyama, M., "Hydrophobic Silica Aerogels,' J. Non-Cryst. Solids, 186, 23 (1995). 